\title{
Intraocular irrigating solutions and barrier function of retinal pigment epithelium
}

\author{
Makoto Araie, Minoru Kimura
}

\begin{abstract}
Aim-To study the effect of intraocular irrigating solutions on the barrier property of the retinal pigment epithelium (RPE).
\end{abstract}

Methods-The isolated rabbit RPEchoroid mounted on Ussing-type chambers under short circuit conditions was used. According to a previous study, the inward (from the choroid to the vitreous side) permeability of the tissue to carboxyfluorescein was adopted as a quantitative index of the barrier function of the RPE cells.

Results-Of the three solutions tested, Krebs-Ringer solution, a commercially available glucose glutathione bicarbonate solution (BSS plus), and glucose citrateacetate bicarbonate solution (Opeguard), BSS plus gave a significantly lower permeability $\left(1.1 \times 10^{-6} \mathrm{~cm} / \mathrm{s}\right.$ on average $)$ than Krebs-Ringer solution or Opeguard (1.9 or $1.8 \times 10^{-6} \mathrm{~cm} / \mathrm{s}$ on average, respectively) (unpaired $t$ test with Bonferroni's correction, $p<0.05)$. Since the major chemical difference between BSS plus and the other two solutions is the incorporation of oxidised glutathione (GSSG), the effects of GSSG were studied using solutions having an identical composition to BSS plus, but with various concentrations of GSSG. The solution containing $0.3 \mathrm{mM}$ GSSG gave significantly lower permeability than that without GSSG $\left(1.1 \times 10^{-6} \mathrm{~cm} / \mathrm{s} v 2.0 \times 10^{-6}\right.$ $\mathrm{cm} / \mathrm{s}$ ) (unpaired $t$ test with Bonferroni's correction, $p<0.05$ ).

Conclusion-It was suggested that BSS plus is less harmful to the barrier function of the RPE cells and that GSSG has a beneficial effect on its maintenance.

(Br f Ophthalmol 1997;81:150-153)

Department of Ophthalmology, University of Tokyo School of Medicine, Tokyo, Japan

M Araie

M Kimura

Correspondence to: Makoto Araie, MD, Department of

Ophthalmology, University

of Tokyo School of Medicine,

7-3-1, Hongo, Bunkyo-ku,

113, Tokyo, Japan.

Accepted for publication 14 October 1996
Vitrectomy allowed us to manage otherwise untreatable ocular pathologies, but this technique significantly involves the ocular tissues surrounding the vitreous cavity. Substitution of the vitreous by an artificial intraocular irrigating solution would probably disturb the physiology of the retina and choroid, and thus influences of intraocular irrigating solutions used in vitrectomy on the retinal function are of primary clinical importance.

Many of the previous studies which addressed this problem have examined the function of the sensory retina, using the electroretinogram (ERG), ${ }^{1-6}$ fluorescein angiogram, ${ }^{7}$ or rate of glycolysis and levels of some metabolites ${ }^{8}$ as factors to be compared. Aside from a study by Shirakawa and associates comparing phagocytotic activity of cultured RPE cells in various intraocular irrigating solutions, ${ }^{9}$ there is a relative paucity of information on the effects to retinal pigment epithelium (RPE). RPE cells are directly exposed to the irrigating solution during vitrectomy when the eye is complicated with rhegmatogenous retinal detachment and these cells play an important role in maintaining the physiology of the sensory retina through their metabolic activity and barrier properties. In the present study, we focused attention on the RPE and compared the effects of various intraocular irrigating solutions on the barrier property of the isolated $\mathrm{RPE}$-choroid preparation.

As irrigating solutions, Krebs-Ringer solution, a glucose glutathione bicarbonate solution, BSS plus (Alcon Laboratories, Fort Worth, TX, USA), and a glucose citrateacetate bicarbonate solution, S-MA ${ }_{2}$ (Opeguard, Senju Pharmaceutical Co, Osaka, Japan), were examined. BSS plus and S-MA were chosen because both were commercially available intraocular irrigating solutions widely used for intraocular surgery including vitrectomy. Oxidised glutathione (GSSG), an ingredient of BSS plus reportedly has a beneficial effect on the barrier function of the corneal endothelium and the blood-aqueous barrier. ${ }^{1011}$ Therefore, the effects of GSSG on the barrier property of the isolated RPE-choroid was also studied.

\section{Materials and methods}

TISSUE PREPARATION

Details of tissue preparation have been described in a previous paper. ${ }^{12}$ Briefly, the eye was enucleated immediately after sacrificing adult New Zealand albino rabbits with an overdose of pentobarbitone. The enucleated eye was bisected at the equator and the posterior half was placed in a Petri dish containing Krebs-Ringer solution maintained at $37^{\circ} \mathrm{C}$ and bubbled with $95 \%$ oxygen and $5 \%$ carbon dioxide The eye cup was opened via three meridional incisions and the vitreous and sensory retina were carefully peeled off. The suprachoroidal tissue was separated from the sclera with meticulous care and a rectangular RPE-choroid complex was isolated. The isolated RPE-choroid was then placed between two halves of an Ussing-type chamber according to procedures described previously. ${ }^{12}$ The exposed tissue surface area was $0.14 \mathrm{~cm}^{2}$ and the volume of each chamber was $7.0 \mathrm{ml}$. Both chambers were filled with one of the test solutions bubbled with $95 \%$ oxygen and 5\% 


\begin{tabular}{lccc}
$\begin{array}{l}\text { Table } 1 \\
\text { (mmol/l) }\end{array}$ & Chemical composition of solutions examined \\
\hline Constituent & BSS plus & S-M $A_{2}$ & Krebs-Ringer \\
\hline Sodium chloride & 122.2 & 112.9 & 118.05 \\
Potassium chloride & 5.1 & 4.8 & 4.69 \\
Calcium chloride & 1.0 & 1.2 & 2.15 \\
Magnesium chloride & 1.0 & - & 0.54 \\
Magnesium sulphate & - & 1.2 & - \\
Sodium phosphate & 3.0 & - & 1.01 \\
Sodium bicarbonate & 25.0 & 25.0 & 25.01 \\
Glucose & 5.1 & 8.3 & 11.1 \\
Sodium acetate & - & 4.4 & - \\
Sodium citrate & - & 3.4 & - \\
Oxidised glutathione & 0.3 & - & - \\
pH & 7.4 & 7.4 & 7.4 \\
Osmolality (mOsm) & 306 & 293 & 298 \\
\hline
\end{tabular}

carbon dioxide and the system was maintained at $37^{\circ} \mathrm{C}$. All experiments were carried out under short circuit conditions, wherein the short circuit current (SCC) was continuously monitored and the transepithelial potential difference was measured every 15 minutes throughout the experimental period of about 3 hours. Rabbits were treated according to the ARVO Resolution on the Use of Animals in Research.

MEASUREMENT OF BARRIER PROPERTY OF RPE Since our previous study indicated that the inward (from the choroid to the vitreous side) movement of carboxyfluorescein across the isolated rabbit RPE-choroid occurs almost exclusively by passive diffusion through the paracellular spaces, ${ }^{13}$ this dye was used as a tracer dye for evaluating the barrier property of the RPE. After an approximately 40 minutes of stabilisation of the SCC, reagent grade 5(6)carboxyfluorescein (Eastman Kodak Co, Rochester, NY, USA) was added to the choroidal side chamber to give a final dye concentration of $300 \mu \mathrm{M}$ in the chamber. Thereafter, the inward movement of the dye was determined for 2 hours and the inward tissue permeability to carboxyfluorescein $(\mathrm{cm} / \mathrm{s})$ was calculated as previously described. ${ }^{12}$

INTRAOCULAR IRRIGATING SOLUTIONS

In the first experimental series, Krebs-Ringer solution was freshly prepared in the laboratory just before use, freshly manufactured $\mathrm{S}-\mathrm{MA}_{2}$ (Opeguard) was purchased from Senju Pharmaceutical Co Osaka, and BSS plus was kindly supplied by Santen Pharmaceutical Co, Osaka. The chemical compositions of these solutions are listed in Table 1. The inward permeability of the isolated rabbit RPE-choroid to carboxyfluorescein was determined using each of these three solutions and the results were compared.

Table 2 Comparison of BSS plus, S-MA $A_{2}$, and Krebs-Ringer solution

\begin{tabular}{llll}
\hline Solution & $\begin{array}{l}\text { Transepithelial potential } \\
\text { difference }(\mathrm{mV})\end{array}$ & \begin{tabular}{l} 
Tissue resistance $_{\left(\Omega \mathrm{cm}^{2}\right)}$ \\
\hline BSS plus
\end{tabular} S-MA $_{2}$ & $\begin{array}{l}\text { Permeability } \\
\left(\times 10^{-6} \mathrm{~cm} / \mathrm{s}\right)\end{array}$ \\
Krebs-Ringer solution $^{*}$ & $114(16)$ & $5.8(1.2)$ & $1.05(0.16) \dagger \ddagger$ \\
\hline
\end{tabular}

Values are mean (SD) in seven experiments.

${ }^{\star}$ Permeability of isolated RPE-choroid to carboxyfluorescein.

†Between group difference was significant at $\mathrm{p}<0.01$ (unpaired $t$ test with Bonferroni's correction for multiple comparison)

$\ddagger$ Between group difference was significant at $\mathrm{p}<0.05$ (unpaired $t$ test with Aspin-Welch’s correction for unequal variance and Bonferroni's correction for multiple comparison).
In the second experimental series, solutions having the identical chemical composition to BSS plus, except that oxidised glutathione (GSSG) was omitted (BSS plus without GSSG) and a GSSG concentration of $0.1 \mathrm{mM}$ (BSS plus with $0.1 \mathrm{mM}$ GSSG) was added were also generously prepared and supplied by Santen Pharmaceutical Co. The $\mathrm{pH}$ and osmolality of BSS plus without GSSG or BSS plus with $0.1 \mathrm{mM}$ GSSG was 7.4 and 303 mOsm, respectively. The inward tissue permeability was determined using BSS plus, BSS plus with $0.1 \mathrm{mM} \mathrm{GSSG}$, or BSS plus without GSSG and the result was compared to evaluate the effect of the GSSG concentration added.

\section{Results}

COMPARISON AMONG KREBS-RINGER SOLUTION, $S-\mathrm{MA}_{2}$, AND BSS PLUS

The addition of carboxyfluorescein at $300 \mu \mathrm{M}$ caused no significant effects on osmolality or $\mathrm{pH}$ of the solutions. The SCC reached a steady state in 30 minutes and the addition of carboxyfluorescein showed no significant effect on the SCC. The transepithelial potential difference and the tissue resistance showed no significant change during the experiment and no significant difference was seen in the transepithelial potential difference or the tissue resistance among the three solutions (Table 2). The obtained inward permeabilities are summarised in Table 2. The inward permeability obtained with BSS plus was significantly lower than that obtained with Krebs-Ringer solution

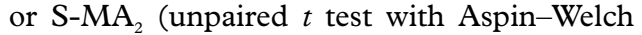
correction for unequal variance and Bonferroni's correction for multiple comparison, $\mathrm{p}<0.05)$. There was no significant difference between the inward permeability obtained with Krebs-Ringer solution and that obtained with $\mathrm{S}-\mathrm{MA}_{2}$.

EFFECT OF GSSG CONCENTRATION ADDED

The transepithelial potential difference and the tissue resistance showed no significant change during the experiment and no significant difference was seen in the transepithelial potential difference or the tissue resistance among the three solutions (Table 3). The inward permeabilities obtained are summarised in Table 3. The inward permeability obtained with BSS plus was significantly lower than that obtained with BSS plus without GSSG (unpaired $t$ test with Aspin-Welch correction for unequal variance and Bonferroni's correction for multiple comparison, $\mathrm{p}<0.05$ ), while it showed no significant difference from that obtained with BSS plus with 0.1 mM GSSG.

\section{Discussion}

According to our previous study, ${ }^{13}$ unlike the isolated dog RPE-choroid, ${ }^{14}$ determination of the inward permeability to carboxyfluorescein in the isolated rabbit RPE-choroid is little affected by the outward (from the vitreous to the choroid side) active transport of carboxyfluorescein, and the inward permeability obtained using the present preparation is thought to almost exclusively reflect the diffusion of the 
dye through paracellular spaces. The rabbit is cheaper to obtain and better suited for extensive studies. Further, since the effects of intraocular irrigating solutions on the barrier function of the corneal endothelium and the blood-aqueous barrier (BAB) have also been studied in rabbit eyes, the results obtained for the RPE can be directly compared with those obtained for the corneal endothelium and the $\mathrm{BAB}$ in the same species. In the present study, the transepithelial potential difference presently recorded was 4-5 $\mathrm{mV}$ (retinal side positive) and lower than those recorded in the $\mathrm{RPE}$-choroid sclera preparation from the rabbit, ${ }^{15}$ while the transepithelial electrical resistance was similar to that reported for the RPE-choroid preparations from $\operatorname{dog}^{16}$ or sheep. ${ }^{17}$ These results suggest that the present experimental condition may not be a perfect replication of the in vivo state of functioning of the RPE cells but the damage, if it existed, would not be serious, and that the present preparation can be used for studying effects of various intraocular irrigating solutions, as long as comparisons are carried out under the same conditions.

BSS plus or another glucose glutathione bicarbonate solution, glutathione bicarbonate Ringer (GBR), has been consistently found to be less harmful than $\mathrm{S}-\mathrm{MA}_{2}$ for the corneal endothelial cells ${ }^{18-21}$ or the BAB. ${ }^{11}$ However, the results obtained for these solutions in the retina or RPE were somewhat different; S-MA $\mathrm{M}_{2}$ was found to be better in maintaining ERG than GBR in rabbits ${ }^{2}$ or than BSS plus in patients. ${ }^{6}$ Phagocytotic activity of the cultured RPE cells was also reported to be better maintained in S-MA ${ }_{2}$ than in BSS plus. ${ }^{9}$ In contrast with these previous studies, the present result suggested that BSS plus is safer for the barrier function of the RPE cells than $\mathrm{S}-\mathrm{MA}_{2}$ or Krebs-Ringer solution, giving a lower permeability of the isolated RPE-choroid to carboxyfluorescein. In the previous studies, where the effects of irrigation with BSS plus, S-MA other solutions on the corneal endothelial permeability $^{18}$ or the $\mathrm{BAB}$ permeability ${ }^{11}$ were compared in rabbits, BSS plus was found to have the least damage on the barrier properties of these ocular tissues. The result obtained here on the isolated rabbit RPE-choroid agrees well with those of the above studies, suggesting that BSS plus is a safe commercially available irrigating solution for the intercellular junctions of ocular tissues.

Chemically, BSS plus, S-MA, and KrebsRinger solution differ in several aspects: (1) $\mathrm{S}-\mathrm{MA}_{2}$ or Krebs-Ringer solution lacks oxidised

Table 3 Effect of oxidised glutathione (GSSG) concentration in solution

\begin{tabular}{llll}
\hline Solution & $\begin{array}{l}\text { Transepithelial potential } \\
\text { difference }(\mathrm{mV})\end{array}$ & $\begin{array}{l}\text { Tissue resistance } \\
\left(\Omega \mathrm{cm}^{2}\right)\end{array}$ & $\begin{array}{l}\text { Permeability } \\
\left(\times 10^{-6} \mathrm{~cm} / \mathrm{s}\right)\end{array}$ \\
\hline BSS plus (0.3 mM GSSG) & $142(39)$ & $4.4(1.1)$ & $1.08(0.17) \dagger$ \\
BSS plus (0.1 mM GSSG) & $119(33)$ & $5.1(0.6)$ & $1.22(0.20)$ \\
BSS plus (without GSSG) & $123(35)$ & $4.2(1.4)$ & $2.00(0.70) \dagger$ \\
\hline
\end{tabular}

Values are mean (SD) in seven experiments.

*Permeability of isolated RPE-choroid to carboxyfluorescein.

†Between group difference was significant at $\mathrm{p}<0.05$ (unpaired $t$ test with Aspin-Welch's correction for unequal variance and Bonferroni's correction). glutathione (GSSG), while BSS plus contains it at a concentration of $0.3 \mathrm{mM}$; (2) $\mathrm{S}-\mathrm{MA}_{2}$ contains about $4 \mathrm{mM}$ of citrate and acetate, but these are absent in BSS plus and Krebs-Ringer solution, and (3) S-MA 2 lacks phosphate, while the other two solutions contain it. The second and third differences do not seem to be responsible for the presently observed discrepancy among the three tested solutions, since the isolated RPE-choroid showed a similar permeability to carboxyfluorescein in S-MA $\mathrm{MA}_{2}$ and Krebs-Ringer solution.

To examine the possibility that the observed difference was mainly attributable to the presence or absence of GSSG in the solution, the permeability was determined using three solutions containing various concentrations of BSS plus: commercially available BSS plus, BSS plus without GSSG, or BSS plus with $0.1 \mathrm{mM}$ GSSG. The permeability obtained using BSS plus without GSSG averaged $2.0 \times 10^{-6} \mathrm{~cm} / \mathrm{s}$. This value was similar to that obtained using S-MA $\mathrm{MA}_{2}$ or Krebs-Ringer solution, 1.8 and $1.9 \times$ $10^{-6} \mathrm{~cm} / \mathrm{s}$, respectively, but significantly greater than that obtained using BSS plus. On the other hand, the value obtained using BSS plus with $0.1 \mathrm{mM}$ GSSG was not significantly different from that obtained using BSS plus. These findings suggest that as far as the permeability of the isolated RPE-choroid to carboxyfluorescein is concerned, absence of GSSG was mainly responsible for the higher value obtained using S-MA $\mathrm{A}_{2}$ or Krebs-Ringer solution.

Although the basis for the currently observed effect of GSSG remains conjectural, it may be related to the glutathione redox system which is involved in the protection of $-\mathrm{SH}$ groups in enzymes and membranes. In ocular tissues, it was reported that the redox state of glutathione in the corneal endothelial cells played a role in the maintenance of its barrier function. ${ }^{22}$ Riley reported that a glutathione reductase inhibitor, BCNU, in the medium damaged the barrier function of the corneal endothelium rather than its active transporting function and that the addition of reduced glutathione, GSH, but not GSSG, protected the observed damage. ${ }^{23}{ }^{24}$ Anderson et al reported that exogenous GSSG rather than GSH is responsible for the increased corneal deturgescence and the effect of BCNU was inhibited by perfusion with a solution containing $0.1 \mathrm{mM}$ GSSG. ${ }^{25}{ }^{26}$ Cultured human RPE cells contain $\mathrm{GSH},{ }^{27}$ and glutathione peroxidase activity is present in the rabbit RPE cells. ${ }^{28}$ Exogenous $\mathrm{GSH}$ of $0.01 \mathrm{mM}$ or higher is reported to provide protection for cultured human RPE cells against oxidative injury. ${ }^{29}$ Although its exact mechanism is unclear at the present time, it may be possible that GSSG in the intraocular irrigating solution exerted a beneficial effect on the intracellular redox state of glutathione and consequently a beneficial effect on the maintenance of the barrier property of the RPE cells.

Surgical outcome of vitrectomy is determined by many factors and the effect of the barrier function of the RPE cells on it is certainly only minimal, if any. The corneal endothelium irrigated with $\mathrm{S}-\mathrm{MA}_{2}$ showed 
$20 \%$ higher permeability to carboxyfluorescein than that irrigated with BSS plus. ${ }^{18}$ In a randomised clinical trial, BSS plus was found to cause significantly less corneal swelling on the first postoperative day than did $\mathrm{S}-\mathrm{MA}_{2}$ in eyes that had undergone extracapsular cataract extraction with posterior chamber lens implantation. ${ }^{21}$ Further, the eyes operated using $\mathrm{S}-\mathrm{MA}_{2}$ showed significantly more postoperative loss and deterioration of morphological characteristics in the corneal endothelial cells. ${ }^{21}$ These findings in the above cited clinical study suggest that $20 \%$ deterioration of the barrier property may imply not only change in the intercellular structures, but also considerable damage in the cell function itself, as far as the corneal endothelium is concerned. In the present experiment, the RPE cells irrigated with $\mathrm{S}-\mathrm{MA}_{2}$ or BSS plus without GSSG showed $70 \%$ higher permeability to carboxyfluorescein than those irrigated with BSS plus with GSSG. The above results obtained in the corneal endothelium suggest that irrigation with these GSSG-free solutions may cause considerable damage not only to the bloodocular barrier, but also to other physiological functions of the RPE cells. The use of BSS plus may be preferable and safer, especially in eyes where subretinal procedures are needed during vitrectomy.

1 Moorehead LC, Redburn DA, Merrit J, Garcia CA. The effects of intravitreal irrigation during vitrectomy. $A m \mathcal{F}$ Ophthalmol 1979;88:239-45.

2 Negi A, Honda Y, Kawano S. Effects of intraocular irrigating solutions on the electroretinographic b-wave. Am $\mathcal{f}$ Ophthalmol 1981;92:28-37.

3 Honda Y. Biological basis of vitreous surgery. Acta Soc Ophthalmol fpn 1981;85:1910-49.

4 Kim SY, Nao-i N, Honda Y. Effects of low molecular weight dextran in intraocular irrigating solutions on ERG B-wave. Metab Pediatr Syst Ophthalmol 1985;8:166-8.

5 Horiguchi M, Miyake Y. Effect of temperature on electroretinograph readings during closed vitrectomy in humans. Arch Ophthalmol 1991;109:1127-9.

6 Maruiwa F, Kim S-D, Nao-i N, Sawada A. Effect of intraocular irrigating solution on electroretinogram during intraocular irrigating solution on electroretinogram during pars plana

7 Alarez MAS, Jimeno JCP. Role of the intraocular irrigating solutions in the pathogenesis of the postvitrectomy retinal edema. Curr Eye Res 1987;6:1369-79.

8 Winkler BS. Comparison of intraocular solutions on glycolysis and levels of ATP and glutathione in the retina. $f$ Cataract Refract Surg 1988;14:633-7.

9 Shirakawa $\mathrm{H}$, Matsumura $M$, Honda Y, Tsukahara I, Ogino $\mathrm{N}$, Nagata $M$. Effects of intraocular irrigating solutions on phagocytosis of latex particles by cultured chick retinal pigment epithelial cells. Acta Soc Ophthalmol fpn 1983;87: $415-21$

10 Araie M, Shirasawa E, Hikita M. Effect of oxidized glutathione on the barrier function of the corneal endothelium. Invest Ophthalmol Vis Sci 1988;29:1884-7.

11 Araie $M$, Shirasawa E, Ohashi T. Intraocular irrigating solutions and permeability of the blood-aqueous barrier. Arch Ophthalmol 1990;108:882-5.

12 Koyano S, Araie M, Eguchi S. Movement of fluorescein and its glucuronide across retinal pigment epithelium-choroid. Invest Ophthalmol Vis Sci 1992;34:531-8.

13 Kimura M, Araie M, Koyano S. Movement of carboxyfluorescein across retinal pigment epithelium-choroid. Exp Eye Res 1996;63: 651-6.

14 Tsuboi S, Pederson JE. Permeability of the isolated dog pigment epithelium to carboxyfluorescein. Invest Ophthalmol Vis Sci 1986;27:1767-70.

15 Frambach DA, Valentine JL, Weiter JJ. Initial observation of rabbit retinal pigment epithelium-choroid-sclera preparations. Invest Ophthalmol Vis Sci 1988;29:814-7.

16 Tsuboi S, Manabe R, Iizuka S. Aspects of electrolyte transport across isolated dog retinal pigment epithelium. $A m \mathcal{F}$ Physiol 1986;250: F781-4.

17 Pascuzzo GJ, Johnson JE, Pautler EL. Glucose transport in isolated mammalian pigment epithelium. Exp Eye Res 1980;30:53-8.

18 Araie M. Barrier function of corneal endothelium and the intraocular irrigating solutions. Arch Ophthalmol 1986;104: $435-8$.

19 Glasser DB, Matsuda M, Ellis JG, Edelhauser HF. Effects of intraocular irrigating solutions on the corneal endothelium after in vivo anterior chamber irrigation. Am $\mathcal{f}$ Ophthalmol 1985;99:321-8.

20 Edelhauser $\mathrm{HF}$. Intraocular irrigating solutions. In: Lamberts DW, Potter DE, eds. Clinical ophthalmic pharmacology. 1st ed. Boston: Little, Brown, 1987:431-44.

21 Matsuda M, Kinoshita S, Ohashi Y, Shimomura Y, Ohguro $\mathrm{N}$, Okamoto $\mathrm{T}$, et al. Comparison of the effects of intraocular irrigating solutions on the corneal endothelium in intraocular lens implantation. Br f Ophthalmol 1991;75: $476-9$

22 Edelhauser HF, Van Horn DL, Miller P, Pederson HJ. Effect of thiol-oxidation of glutathione with diamide on corneal endothelial function, junctional complexes, and microfilaments. F Cell Biol 1976;68:567-78.

23 Riley MV. A role for glutathione and glutathione reductase in control of corneal hydration. Exp Eye Res 1984;39:7518.

24 Riley M. Pump and leak in regulation of fluid transport in rabbit cornea. Curr Eye Res 1985;4:371-6.

25 Anderson EI, Fischbarg J, Spector A. Disulfide stimulation of fluid transport and effect on ATP level in rabbit corneal endothelium. Exp Eye Res 1974;19:1-10.

26 Anderson EI, Wright DD. The roles of glutathione reductase and $\gamma$-glutamyl transpeptidase in corneal transendothelial fluid transport mediated by oxidized glutathione and glucose. Exp Eye Res 1982;35:11-9.

27 Reddy VN, Lin L-R, Giblin FJ, Chakrapani B, Yokoyama T. Study of the polyol pathway and cell permeability changes in human lens and retinal pigment epithelium in tissue culture. Invest Ophthalmol Vis Sci 1992;33:2334-9.

28 Ostrobsky MA, Sakina NL, Dontsov AE. An antioxidative role of ocular screening pigments. Vision Res 1987;27:893-

29 Sternberg Jr P, Davidson PC, Jones DP, Hagen TM, Reed $\mathrm{RL}$, Drews-Botsch C. Protection of retinal pigment epithelium from oxidative injury by glutathione and precursors. Invest Ophthalmol Vis Sci 1993;34:3661-8. 